\title{
Imaging of Rat Head under Ischemic Conditions by Near-Infrared Computed Tomography
}

\author{
Rentaro ABUmI, ${ }^{1, *}$ Katsuya NAGAI, ${ }^{1}$ Yasunobu ITOH, ${ }^{2}$ Ichiro OdA, ${ }^{2}$ \\ Michinosuke TAKADA, ${ }^{2}$ Mamoru TAMURA, ${ }^{3}$ and \\ Hachiro NAKAGAWA ${ }^{1}$ \\ ${ }^{1}$ Division of Protein Metabolism, Institute for Protein Research, \\ Osaka University, Suita 565, Japan \\ ${ }^{2}$ Analytical Instruments Research Laboratory, Shimadzu Corporation, \\ Nakagyo-ku, Kyoto 604, Japan \\ ${ }^{3}$ Biophysics Division, Research Institute of Applied Electricity, \\ Hokkaido University, Sapporo 060, Japan
}

(Received July 28, 1991)

\begin{abstract}
Summary Light in the near-infrared range $(700-1,200 \mathrm{~nm})$ penetrates living tissues including the skull. Oxy- and deoxy-hemoglobin contents in living tissue can be measured from the absorbance in this range. Myoglobin and cytochrome oxidase also have their absorbance in this range. Therefore, we carried out near-infrared computed tomography by a modification of the method for X-ray CT for humans. Three semiconductor lasers were used as light sources with a laser beam scanner. The transmitted light was measured with a linear detector equipped with 25 photomultipliers. With this device, we constructed images of the heads of anesthetized rats from the differences between the absorbance data at 780 and $805 \mathrm{~nm}$. The brightness of the head images increased in rats with ischemia induced by occlusion of four vessels (carotid). The ischemic area in the central part of the head was shown on exchange transfusion of the blood with perfluorochemical emulsion.
\end{abstract}

Key Words: semiconductor laser, near-infrared spectrophotometry, functional image, hemoglobin oxygenation, CT

X-ray CT and magnetic resonance imaging (MRI) have been widely used to obtain anatomical information on organs in situ. Research to extend MRI to provide information about biological function is currently in progress [1]. Positron emission tomography is at present the best method available for obtain-

\footnotetext{
${ }^{*}$ To whom correspondence should be addressed.
} 
ing functional information. However, there are several problems involved in use of this tomography: its safety is uncertain and the time of measurements is limited, because nuclear tracers are used as probes and these must be prepared in a hospital-based cyclotron before the measurements.

Near-infrared light in the range of 700 to $1,200 \mathrm{~nm}$ penetrates living tissue; and thus spectrophotometry in this wavelength range has been used for a non-invasive quantitative measurement of the oxy- and deoxyhemoglobins in living tissues [24]. We developed an instrument for monitoring the hemoglobin oxygenation of the brain, and three semiconductor lasers of different wavelengths $(780,805$, and $830 \mathrm{~nm}$ ) were used as light sources in the instrument $[4,5]$. Our research suggested that it might be possible to develop a new type of computed tomography using near-infrared light for real-time imaging of changes such as those in the regional distribution of blood and oxygen supply in living tissues. To examine this possibility, we constructed a near-infrared computed tomography (NIR-CT) system and tested it with phantoms containing absorptive and diffusive materials [5, 6]. We succeeded in obtaining images of the phantoms from the absorbance data by NIR-CT and found that the least spatial resolution was less than $10 \mathrm{~mm}$. We also obtained NIR-CT images of the abdomen of anesthetized rats.

In the present study, we obtained NIR-CT images of the head of anesthetized rats and showed the change of images under ischemic conditions induced by the 4-vessel occlusion method established by Pulsinelli and Brierley [7]. We also showed that the ischemic area was intensified by exchange transfusion with a blood substitute, perfluorochemical emulsion (FC-43 Emulsion, The Green Cross, Co., Osaka). This paper reports our procedure and results.

\section{MATERIALS AND METHODS}

A medical fan beam X-ray CT system was modified to construct the NIR-CT system (NIRCT-1000, Shimadzu, Co., Kyoto), as shown in Fig. 1 [5, 6]. In this system, three semiconductor lasers of different wavelengths $(780,805$, and $830 \mathrm{~nm})$ and a laser beam scanner were used instead of an X-ray tube. The laser beam was scanned by a scanner mirror. The lasers were in the continuous wave mode, and the light beams were emitted in turn by the time-sharing mode so that the duration of illumination was controlled to be $9.8 \mathrm{~ms}$ at each illuminated spot $\left(3 \mathrm{~mm}^{2}\right)$. The powers at illuminated spots varied depending on the wavelength $(18,54$, and 162 $\mathrm{mW}$ at 780,805 , and $830 \mathrm{~nm}$, respectively). The transmitted light was detected by a linear detector equipped with 25 glass fibers each of which guided the light to 25 separate photomultipliers. Values of absorbances were calibrated by use of four kinds of glass filters whose absorbances were known at the three wavelengths. The absorbances of the 4 filters were between 4.99 OD and 6.873 OD. Photoelectrons emitted by the light passed through the filters were measured at each photomultiplier. Three simple equations for photoelectrons and values of absorbances were made at the three wavelengths by simple approximation. The absorbances at 2 or 


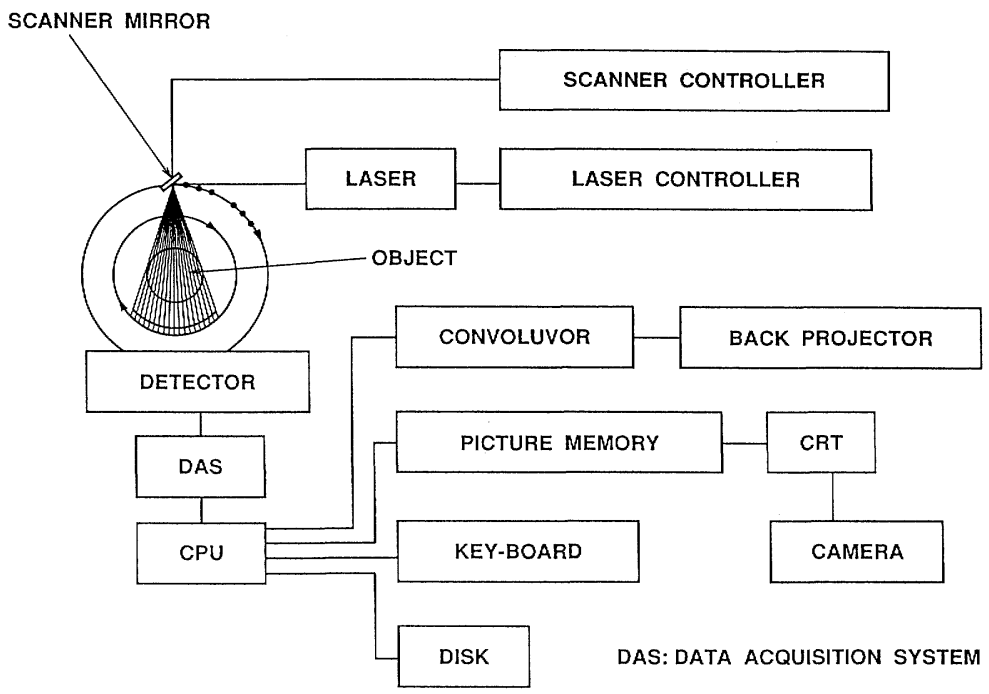

Fig. 1. Block diagram of NIR-CT system (reprinted from reference [6]). The light sources (scanner mirror and lasers) were controlled by a scanner controller and a laser controller. Projection data were detected by detectors and introduced into the CPU (central processing unit) through the DAS (data acquisition system). The data arranged in the $\mathrm{CPU}$ were introduced to a convoluvor and a back projector. The image obtained was displayed on a CRT through a picture memory and saved in a disket file.

3 different wavelengths were measured simultaneously by these equations. The data were obtained at every 6-degree step of rotation, and thus 60 measurements were made in one rotation. The data processing was based on the back projection method used in the X-ray CT system (SCT-2000T-20, Shimadzu, Co., Kyoto). It took $10 \mathrm{~min}$ to obtain all the data in one rotation, and $3 \mathrm{~min}$ to construct one image. The sample less than $100 \mathrm{~mm}$ in diameter could be measured.

Male Wistar rats, weighing 400-430 g, were anesthetized with pentobarbital (50 mg/kg, i.p., Nembutal Injection, Dinabott, Osaka), and femoral arterial and venous catheters were inserted through openings in both lower legs. The hair of the head was shaved to reduce light reflection at the surface. The vertebral arteries on both sides were occluded by electrocauterization and 2 cotton threads were loosely placed around each common carotid artery according to the procedure of Pulsinelli and Brierley [7]. After tracheotomy the trachea was intubated with a tracheal tube, which was connected to a Y-shaped tube. Each rat was immobilized in the supine position on the sample stage by vinyl adhesive tapes so that measurement of a coronal section including the mid-points of two lines, each connecting the eye and external acoustic meatus of one side, could be made. The muscles were paralyzed with $50 \mu \mathrm{g}$ of pancuronium bromide (Mioblock, Organon, Tokyo), and the animal was artificially ventilated with an animal ventilator using mixed gas $\left(33 \% \mathrm{O}_{2}\right.$ and $67 \% \mathrm{~N}_{2} \mathrm{O}$ ). The ventilation was adjusted to maintain $\mathrm{PaCO}_{2}$ and $\mathrm{PaO}_{2}$ 
between 30 to $40 \mathrm{mmHg}$ and about $100 \mathrm{mmHg}$, respectively. After the first measurement by NIR-CT was completed, an ischemic condition was induced by tightening of the threads around the two carotid arteries, which resulted in 4-vessel occlusion. The second measurement was made $10 \mathrm{~min}$ after induction of ischemia.

The blood of the rat with cerebral ischemia was then replaced with FC-43 Emulsion by exchange transfusion using a roller pump. The inspired gas was switched to $100 \% \mathrm{O}_{2}$ when the exchange transfusion was started. The ventilation was adjusted to maintain $\mathrm{PaCO}_{2}$ and $\mathrm{PaO}_{2}$ between 30 to $40 \mathrm{mmHg}$ and above 350 $\mathrm{mmHg}$, respectively. The transfusion was stopped when the remaining hemoglobin decreased to less than $10 \%$ of the initial control level. The speed of transfusion was $1.5-2.5 \mathrm{ml} / \mathrm{min}$. Total exchange volume was $60-100 \mathrm{ml}$. The third measurement was made after the completion of exchange transfusion. After the completion of experiments, we observed blood that remained in the brain and muscles in the central part of the head by dissecting the rats. The NIR-CT images were constructed on the basis of the differences between the absorbance data at 780 and $805 \mathrm{~nm}$.

\section{RESULTS}

Figure 2 shows the optical density of a head of a rat at each wavelength obtained by each photomultiplier in one step of rotation of the NIR-CT. Figure 3 shows the positions of the head of the rat, photomultipliers, and the light source used in the measurement of Fig. 2. The data were obtained under the directional conditions when the light source was on the left-hand, and the detector was on the right-hand, side of the rat. Figure 3(b) is a schematic view of the rat seen from below. Four photomultipliers, No. 12-No. 15, detected the light that passed through the head of the rat, as shown in Fig. 3(a). The photomultipliers of No. 1No. 11 and No. 16-No. 25 registered 0 OD, because they were off the head of the rat. The absorbance at each photomultiplier at each wavelength decreased in the order 780, 805, $830 \mathrm{~nm}$ (Fig. 2(a), (b), and (c)). After the induction of ischemia, the absorbances at $780 \mathrm{~nm}$ increased at photomultipliers No. 13-No. 15 and decreased at No. 12 (Fig. 2(a)). The absorbances at 805 and $830 \mathrm{~nm}$ decreased at photomultipliers No. 12 and No. 13, changed little at No. 14, and increased at No. 15 after the induction of ischemia (Fig. 2(b) and (c)). The datum values obtained by subtraction of the absorbance at $805 \mathrm{~nm}$ from that at $780 \mathrm{~nm}$ increased at photomultipliers No. 13-No. 15, and decreased at No. 12 after the induction of ischemia (Fig. 2(d)). These changes in absorbances were explained by the deoxygenation of hemoglobin and myoglobin and the reduction of cytochrome oxidase (photomultiplier No. 13-No. 15), and by the increase (photomultiplier No. 15) and decrease (photomultiplier No. 12 and No. 13) of hemoglobin content, because the wavelength of $805 \mathrm{~nm}$ is an isobestic point of hemoglobin. There was a trough at photomultiplier No. 13 under the control and ischemic conditions in absorbance data obtained by subtraction (Fig. 2(d)), which corresponded to the dark area in the central part of the head as seen in Fig. 4(a) and (b). After the completion of 


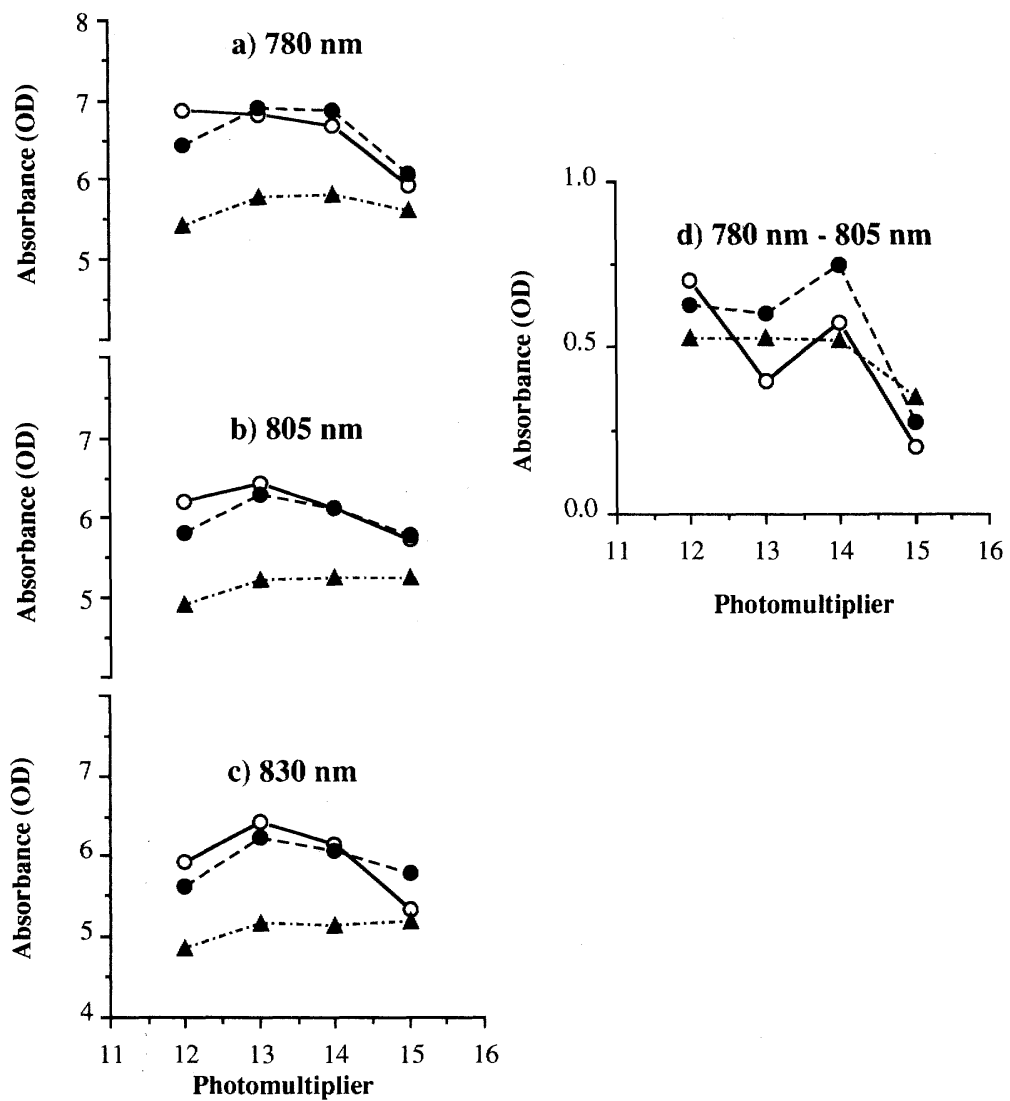

Fig. 2. Absorbance data of the head of a rat at each photomultiplier at each wavelength in one step of rotation of the NIR-CT. The data were obtained in the same direction when the light source was on the left-hand, and the detector was on the right-hand, side of the rat. Four photomultipliers, No. 12-No. 15, detected the light that passed through the head of the rat, and are shown in the horizontal axis. $-0-$, Control; ----, $10 \mathrm{~min}$ after 4-vessel occlusion; -- - - after the completion of exchange transfusion with FC-43 Emulsion in addition to 4-vessel occlusion.

the exchange transfusion with FC-43, the absorbances at the three wavelengths decreased as a whole (Fig. 2(a), (b), and (c)), and the differences between the absorbance at 780 and $805 \mathrm{~nm}$ decreased a little (Fig. 2(d)). This means that hemoglobin and myoglobin remained in the rat head even when blood hemoglobin disappeared in some parts of the head after the exchange transfusion.

Figure 4 shows the NIR-CT images of the head of a rat under the control (Fig. 4(a)), ischemic condition (Fig. 4(b)), and that after the exchange transfusion with FC-43 Emulsion under the ischemic condition (Fig. 4(c)). The brain in the dorsal part and muscles in the lateral and ventral parts of the head were observed as bright areas, though left lateral muscles overlapped with the brain. Bones and 


\section{a)}

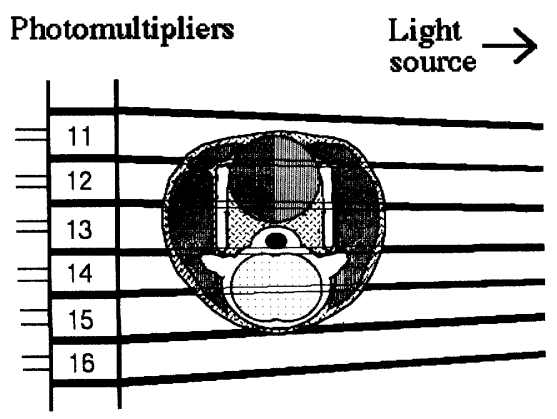

b)

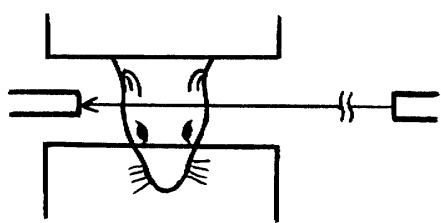

Fig. 3. The positions of the head of the rat, the detector, and the light source in the measurement of Fig. 2. Four photomultipliers, No. 12-No. 15, detected the light that passed through the head of the rat. The photomultipliers No. 1-No. 11 and No. 16-No. 25 were off the head of the rat. (a) Schematic view of head seen from caudal side. (b) Schematic view of head seen from below.

other tissues with low blood concentration were observed as dark areas. The brightness of the image of the ischemic head was higher than that of the control as a whole. A distinction between brain and muscles was not definite in images under the ischemic condition, because the medial part of the head became brighter. After the exchange transfusion, the brightness became lower in the lateral part of muscles of the head (Fig. 4(c)) in which the circulation was still maintained under the ischemic condition and then the blood was replaced by FC-43 Emulsion, because the absorbance of FC-43 is lower than that of blood hemoglobin. The brightness did not change in the ischemic area of the head in which the circulation was arrested.

Figure 5 shows a CT image obtained by the X-ray CT and schemas of a coronal section of the head of a rat at the center between eyes and external acoustic meatus. Figure 5(a) shows the image of the head of a rat obtained by the X-ray CT (SCT-2000T-20, Shimadzu, Co., Kyoto). Bright areas of the skull and mandible are observed in this image. Figure 5(b) shows a schema of a coronal section at this level. Muscles of mastication are located lateral to the skull and the mandible, and ventral to the skull between the bilateral rami mandibulae. Brain is in the skull. Figure 5(c) shows a schema of the image taken by NIR-CT. Blood hemoglobin and muscle myoglobin are the major absorptive materials in the range of near-infrared 

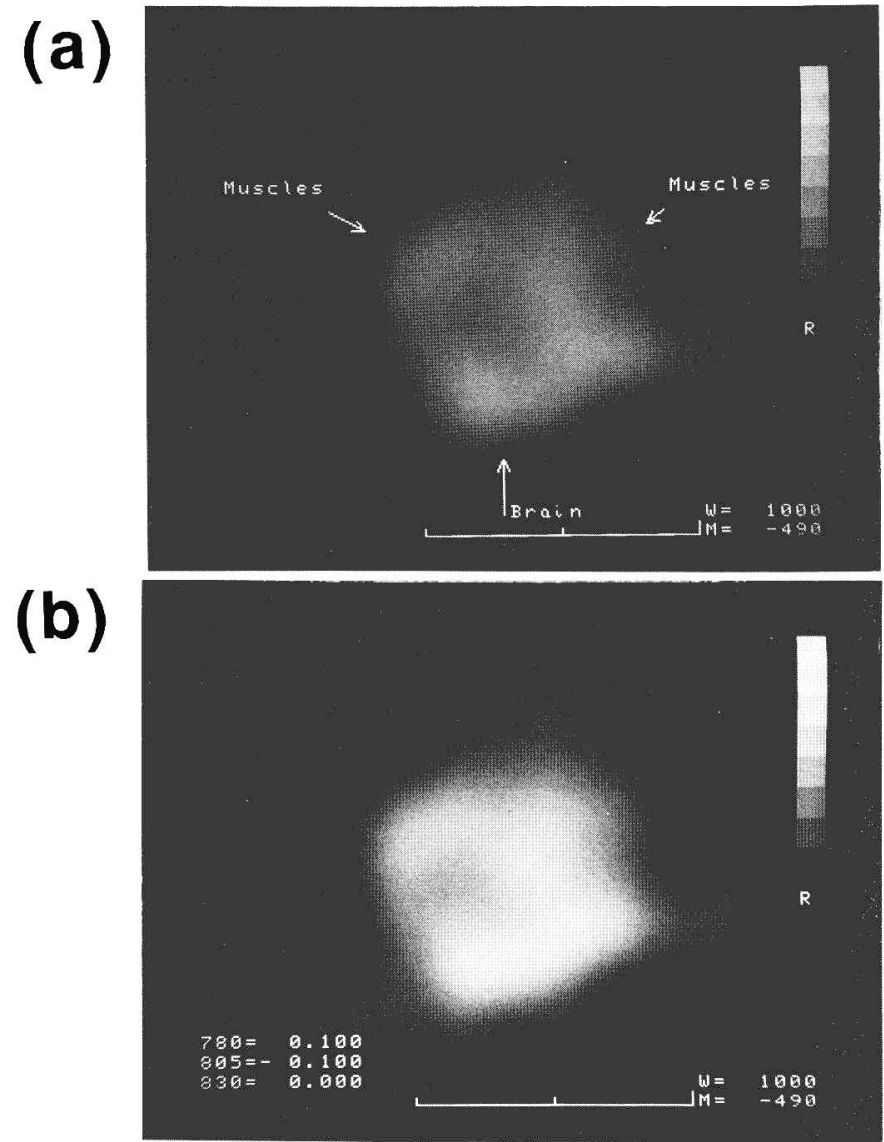

(c)

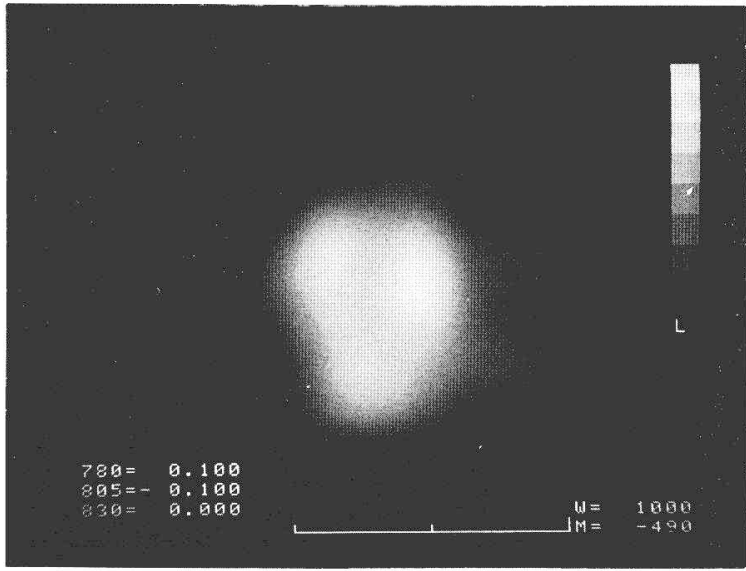

Fig. 4. NIR-CT images of a coronal section of the head of the rat in the supine position at the center between eyes and external acoustic meatus. The scale below each image indicates $10 \mathrm{~mm}$. (a) Control. (b) Ten minutes after 4-vessel occlusion. (c) After the completion of exchange transfusion with FC-43 Emulsion in addition to 4-vessel occlusion.

Vol. 1 1, No. 3, 1991 


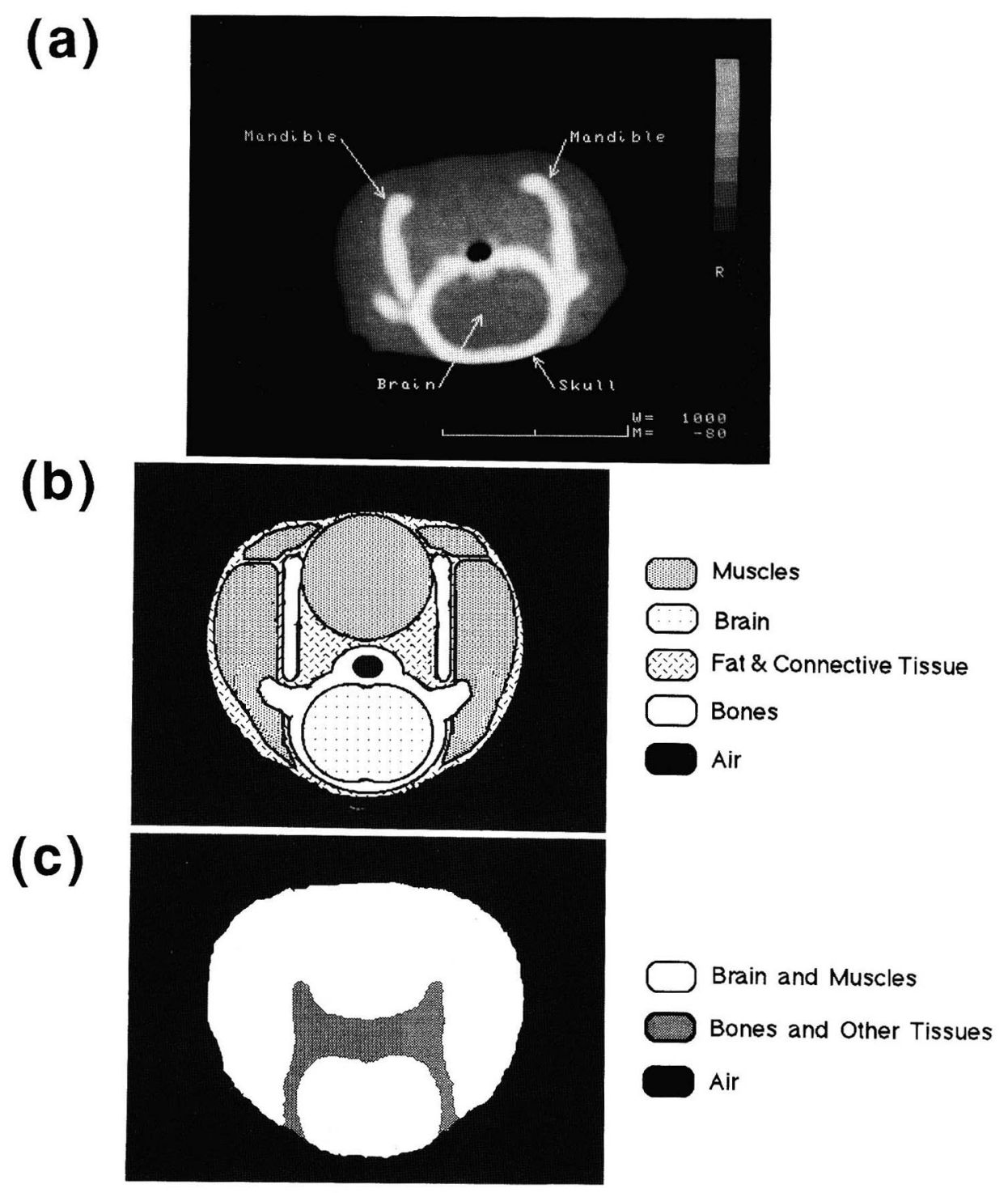

Fig. 5. An image obtained by the X-ray CT and schema of CT images of the head of a rat obtained at the same position as in Fig. 3. (a) Image of the head of a rat obtained by the X-ray CT. (b) Schema of this section. (c) Schema of NIR-CT image.

light. Thus brain in dorsal part, muscles in ventral and lateral parts of the head are observed as bright areas in the image obtained by NIR-CT. Bones are observed as dark areas in the image of the NIR-CT, because they have low absorbance in this wavelength range. There is a tendency that the area with high absorption appears to be bigger than other low absorptive areas in the image of our NIR-CT [5]. Thus 
the brain and muscles appear to be bigger than their real size, and they sometimes overlap in images of our NIR-CT.

\section{DISCUSSION}

In this work we demonstrated that the NIR-CT system that we devised can distinguish bones from brain and muscles, and detect ischemic change in the head of anesthetized rats. This is the first report on imaging by an optical CT system using near-infrared light, and the results suggest its possible use for obtaining information on the physiological functioning of organs or tissues in living animals.

In the measurements by near-infrared spectrophotometry, the absorbance change was assumed to be proportional to the change in the concentration of absorptive materials, and the optical pathlength was assumed to be constant by fixing the position of the light source and the detector [2-4]. But the optical pathlength of near-infrared light is not the same as the shortest route between the light source and the detector, because most of the near-infrared light is scattered and only a small part of it is directly transmitted to the detector through living tissues. The optical pathlength can be estimated from the time of passage of picosecond-length light pulses. Delpy et al. [8] showed that the mean optical pathlength across the rat head was 5.3 times its diameter. Because the light scattering makes it difficult to measure the absorbance in living tissues quantitatively, there are two ways to develop a light CT system with good spatial resolution: one is a new method to select the directly transmitted light component, and the other is a new algorism available for calculation of both scattered and directly transmitted light. Toida et al. [9] chose the former way. They used an optical heterodyne technique for detecting the directly transmitted light component in an optical CT system, in which a coherent laser beam and a highly sensitive photodetecting system were used. They reported CT images of phantoms and chicken. Several authors examined the latter way by computer simulations or fundamental experiments [10], but a new algorism that makes light CT more quantitative has not been made.

Tamura et al. [11] investigated the effect of absorptive material on the range of light spread in a light-scattering solution composed of hemoglobin and milk, and they found that the range was narrower when the concentration of hemoglobin was higher in the mixture [9]. The percentage of light that passed through the short optical pathlength became greater, because the light that passed through the longer optical pathlength was absorbed by hemoglobin in the solution. This phenomenon should increase the percentage of light that passes through the narrow pathway between the light source and the detector. We assume that it is possible to make images by the NIR-CT, because the major part of the nearinfrared light passes through the short optical path length in living tissue of the size of the head of a rat.

Vol. 11, No. 3, 1991 
We constructed the NIR-CT for the purpose of imaging physiological information in living animals. It must obtain all the data in a short time for this purpose. Thus we decided to modify the X-ray CT and applied the back projection method in processing data from our system on the assumption that the light transmission was straight and without calculating the scattered light component. It took 10 min to obtain all the data in one rotation. This is a short enough time to allow studies on physiological functions. Our imaging data were poor in spatial resolution because of the light scattering and small numbers of photomultipliers that obtained the data. The spatial resolution in images of the head of rats is supposed to be of the same degree as that of phantoms (less than $10 \mathrm{~mm}$ ) [5].

Three wavelengths in the near-infrared range were chosen for this NIR-CT system, and they were the same ones as used in another instrument for monitoring hemoglobin oxygenation in the brain $[4,5]$. The equations in the latter case were derived from experiments in which the light source and the detector were attached to the surface of the head where muscles did not exist between them. The absorbances at the three wavelengths were measured in the perfused rat head in which the hematocrit value was varied in order to derive the equations [4], because the absorption spectrum of cytochrome oxidase overlaps with that of hemoglobin. We could not differentiate blood hemoglobin from muscle myoglobin by the absorbance data at the three wavelengths, because their absorption spectra overlap each other and shift in the same pattern according to the oxygenation state at the three wavelengths [11]. Thus it was impossible to derive equations for the NIR-CT to calculate oxygenation of hemoglobin and myoglobin separately, because the light source and detector were removed from the head and there were muscles besides brain between them in the measurement by the NIR-CT.

Light reflection at the surface of the head remained in the measurements, though the hair of the head was shaved to reduce it. It would be less, if some liquid could have been placed between the light source and the head. However, we could not use a liquid because of the rotation of the light source and the detector. Most of the near-infrared light was scattered in the head of the rat, and only a small part of it was transmitted directly to a photomultiplier opposite to the light source. The value of absorbance was calculated as the logarithm of the proportion of light intensity at the photomultiplier to that at the light source. Thus light intensity at the photomultiplier was diminished by absorption in the head as well as by light reflection at the surface and light scattering in the head, and values obtained in the head of the rat were high at the 3 wavelength (Fig. 2(a), (b), and (c)). They were not obtained by incorrect measurements. Calibrations for data acquisition were performed with four kinds of filters whose absorbances were known at the three wavelengths. However, the data obtained by subtraction of the data at $805 \mathrm{~nm}$ from that at $780 \mathrm{~nm}$ were small, and they increased by $0.2 \mathrm{OD}$ in the central part of the head at photomultiplier No. 13 after the induction of ischemia (Fig. 2(d)). These values were of the same degree as those obtained in the rat head by spectrophotometry $[3,4]$. We suppose that the same degree of light reflection and 
scattering occurred in the head at the two wavelengths, and the portion of light reflection and scattering was eliminated from data obtained by subtraction. We also suppose that a dual-wavelength spectroscopic method would be better than the single-wavelength method to detect absorptive materials, though it was not quantitative. Moreover, it was difficult to detect the ischemic change by single wavelength measurement. We used differences between the absorbance data at 780 and $805 \mathrm{~nm}$ to construct NIR-CT images, because differences between absorbance data at 805 and $830 \mathrm{~nm}$ were too small to construct an image. Thus the images of our NIR-CT represented the sum of absorbances of hemoglobin, myoglobin, and cytochrome oxidase in the head of a rat under the control and ischemic conditions.

We exchanged blood for FC-43 in the anesthetized rat under ischemia in order to depict the ischemic area of the head by the NIR-CT. Injection of a dye into the ascending aorta is the usual method to estimate cerebral perfusion in the ischemic head [7]. Our method to examine the arrest of circulation in the ischemic head was not orthodox but meaningful, because it could be carried out in living rats by the NIR-CT.

A part of this study was supported by a Grant-in-Aid for Scientific Research (No. 02557014) from the Ministry of Education, Science, and Culture of Japan.

\section{REFERENCES}

1. Ogawa, S., Lee, T.M., Kay, A.R., and Tank, D.W. (1990): Brain magnetic resonance imaging with contrast dependent on blood oxygenation. Proc. Natl. Acad. Sci. U.S.A., 87, 98689872 .

2. Jöbsis, F.F. (1977): Noninvasive, infrared monitoring of cerebral and myocardial oxygen sufficiency and circulatory parameters. Science, 198, 1264-1267.

3. Hazeki, O., and Tamura, M. (1988): The quantitative analysis of hemoglobin oxygenation state of rat brain in situ as monitored by near-infrared spectrophotometry. J. Appl. Physiol., 64, 796-802.

4. Tamura, T., Eda, H., Takada, M., and Kubodera, T. (1989): New instrument for monitoring hemoglobin oxygenation, in Advances in Experimental Medicine and Biology, Vol. 248, ed. by Rakusan, K., Bilo, G.P., Goldstick, T.K., and Turek, Z., Plenum Press, New York, pp. $103-107$.

5. Oda, I., Ito, Y., Eda, H., Tamura, T., Abumi, R., Nagai, K., Nakagawa, H., and Tamura, M. (1991): Non-invasive hemoglobin oxygenation monitor and computed tomography by NIR spectrophotometry, in Time-Resolved Spectroscopy and Imaging of Tissues, SPIE Vol. 1431, ed. by Chance, B. and Katzir, A., the Society of Photo-Optical Instrumentation Engineers, Washington, pp. 284-293.

6. Ito, Y., Oda, M., Takada, M., Kubodera, T., Nagai, K., Nakagawa, H., and Tamura, M. (1989): An experiment of light beam computed tomography, in Proc. 2nd Internatl. NIRS Conference, ed. by Iwamoto, M. and Kawano, S., Korin Publ., Tokyo, pp. 305-312.

7. Pulsinelli, W.A., and Brierley, J.B. (1979): A new model of bilateral hemispheric ischemia in the unanesthetized rat. Stroke, 10, 267-272.

8. Delpy, D.T., Cope, M., van der Zee, P., Arridge, S., Wray, S., and Wyatt, J.S. (1988): Estimation of optical pathlength through tissue by direct time of flight measurement. Phys. Med. Biol., 33, 1433-1442.

9. Toida, M., Kondo, M., Ichimura, T., and Inaba, H. (1990): Experimental verification of

Vol. 11, No. 3, 1991 
image detection in highly scattering media using antenna properties of optical heterodyne microscope scheme. Electron. Lett., 26, 700-702.

10. Singer, J.R., Grünbaum, F.A., Kohn, P., and Zubelli, J.P. (1990): Image reconstruction of the interior of bodies that diffuse radiation. Science, 248, 990-993.

11. Tamura, M., Nomura, Y., and Hazeki, O. (1987): Laser tissue spectroscopy—near infrared CT. Rev. Laser Eng., 15, 657-665. 